\title{
Key considerations for conducting Chinese medicine clinical trials in hospitals
}

\author{
Johannah L Shergis*, Shefton Parker, Meaghan E Coyle, Anthony L Zhang and Charlie C Xue
}

\begin{abstract}
Conducting clinical trials of Chinese medicines (CM) in hospitals presents challenges for researchers. The success of hospital-based CM clinical trials may be influenced by the protocol design, including the maintenance of CM theory in compliance with scientific rigour and hospital guidelines and justified treatment approaches with results that can translate into clinical practice. Other influences include personnel and resources such as a dedicated team open to CM with an established research culture and the ability to maximise participant recruitment. This article identifies the key challenges and limitations of conducting CM clinical trials in Australian hospitals.
\end{abstract}

\section{Commentary}

Chinese medicine $(\mathrm{CM})$ treatments, including acupuncture, herbal medicine, remedial manual therapy, and exercise therapy (Tai Chi) are growing in popularity [1-3]. At the same time the healthcare community have begun to acknowledge the preferences of patients to receive treatment with $\mathrm{CM}$ in the United Kingdom, United States and Australia [4-7]. In Australia, CM practitioners must be registered nationally by the Chinese Medicine Board of Australia through the Australian Health Practitioners Regulation Agency in order to practice acupuncture and prescribe Chinese herbal medicine [8].

The practice and paradigmatic philosophies of CM differ from those of Western medicine (WM). Traditional CM diagnostic methods consider factors such as the environment, seasons, emotions, diet, and lifestyle. In addition WM diagnostic approaches are also used by $\mathrm{CM}$ practitioners to provide a comprehensive diagnosis and individualised treatment plan. In contrast, WM focuses on pathological processes and uses diagnostic instruments or tests or both to classify illness, and places less of an emphasis on a person's interaction with the environment. These differences may create gaps in communication between the disciplines; however, recently cooperation has grown [9].

Evidence-based medicine (EBM) including randomized controlled trials (RCT) is likely to bridge the gap further

\footnotetext{
* Correspondence: johannah.shergis@rmit.edu.au

Traditional and Complementary Medicine Research Program, School of Health Sciences and Health Innovations Research Institute (HIRi), RMIT University, PO Box 71, Bundoora, Victoria 3083, Australia
}

between CM and WM. Increasing numbers of RCTs are being undertaken to determine the efficacy and safety of CM interventions [10]. CM researchers have been striving to achieve high standards of scientific rigor through RCTs. However, this has presented challenges in harmonizing $\mathrm{CM}$ theory and the RCT design required to enable effective translation [11].

Given the relatively short history of RCTs in CM outside China, a substantial proportion of $\mathrm{CM}$ studies were usually conducted in universities or community clinics. In Australia, hospitals are increasingly involved in clinical studies since the introduction of national strategies and processes to encourage greater engagement and participation of hospitals in research [12]. A search of the Australian and New Zealand Clinical Trials Registry (ANZCTR) on 23 August 2012 identified 144 alternative and complementary medicine RCTs with ethical approval being conducted in Australia. Of these, 71 were $\mathrm{CM}$ studies, and 16 were identified as being conducted in hospitals. The availability of participants (e.g., acute, sub-acute and outpatients), easy access to hospital resources (e.g., functional magnetic resonance imaging, fMRI) and medical expertise are major factors which make hospitals suitable environments for undertaking CM clinical trials.

As a typical example, this paper will highlight some of the challenges of undertaking RCTs in CM interventions, based on our multi-centre hospital studies in Melbourne, Australia (Table 1). The Multiple Emergency Department Acupuncture Trial (MEDACT) evaluated the analgesic effect of acupuncture for patients presenting to the 
Table 1 Characteristics of the MEDACT and GEARS clinical trials

\begin{tabular}{lll}
\hline & MEDACT & GEARS \\
\hline Condition treated & Acute pain; ankle sprain, non-specific low back pain or migraine & COPD \\
Design & RCT & RCT \\
Funding source & Government funding body (NHMRC) & Government funding body (NHMRC) \\
Source of patients & Hospital ED & Community and outpatients \\
Location of patient visits & Hospital ED & Hospital outpatients \\
Number of sites & 4 & 4 \\
Number of participants & 505 & 168 \\
Number of groups & 3 & 2 \\
Intervention & Acupuncture or acupuncture plus standard care (pharmacotherapy) & Panax ginseng (herb) \\
Control & Standard care (pharmacotherapy) & Placebo \\
Treatment duration & 1 treatment & 6 months \\
Follow-up & 48 hours & 6 months \\
Outcomes & 1. Pain and disability scales & 1. Quality of life \\
& 2. Quality of life & 2. Lung function \\
3. Acceptably of treatment and willingness to repeat & 3. Use of relief medication \\
4. Adverse events & 5. Health resource utilization & 4. Exacerbations
\end{tabular}

COPD: Chronic obstructive pulmonary disease, ED: Emergency department, GEARS: Ginseng extract and respiratory symptoms clinical trial, MEDACT: Multiple emergency department acupuncture control trial, NHMRC: National Health and Medical Research Council, RCT: Randomized controlled trial.

emergency department (ED) with low back pain, ankle sprain or migraine [13]. This study was pragmatic in design and allocated participants randomly to one of three groups: acupuncture alone; acupuncture plus pharmacotherapy; and pharmacotherapy alone. Recruitment occurred in four EDs and the primary outcome was pain relief after one hour. The Ginseng Extract and Respiratory Symptoms (GEARS) is ongoing, and is evaluating the efficacy of a herbal medicine in participants with chronic obstructive pulmonary disease (COPD) [14]. This study is a two-arm parallel RCT and participants are recruited through four metropolitan hospitals. This paper will discuss the issues we encountered, including protocol design, personnel and resources, and participant recruitment.

\section{Protocol design}

RCTs should meet standards of rigorous scientific design. RCTs need to incorporate design elements to maintain internal and external validity for generalisability. In Australian hospitals, CM treatments are not prescribed routinely; thus, researchers need to ensure that the trial is implemented in accordance with hospital policies and guidelines without compromising the quality of the research. Furthermore, in order to be clinically relevant, the results of the trial should be translatable to a clinical practice setting.

A major consideration in designing a hospital-based CM $\mathrm{RCT}$ is the study design. Feasible protocols are required to implement and researchers need to consider the appropriateness and justification for complex individualised interventions and non-standard treatments, particularly when using herbal medicines [15].

Another main consideration for CM studies is the justification of intervention. This is determined predominantly through historical use and is usually supported by records with pharmacological and toxicological reports and detailed information of the herbal product's constituents. Acupuncture studies may encounter similar questions regarding evidence base and mechanism of action, to justify its use for a particular health condition.

Traditional CM diagnosis and treatment are based on interview and observation of the patient, and do not require instrumental tests, as in WM. When a WM diagnosis is required to assess eligibility for inclusion in a pragmatic trial, and the CM diagnosis is used only to guide selection of an appropriate treatment for each individual, the trial may be better suited to the hospital setting. For example, the MEDACT study required the WM diagnosis of an ankle sprain, migraine or low back pain [13]. Although a CM diagnosis was not an inclusion criterion, the acupuncturists were able to select appropriate acupuncture points based on CM theory.

In contrast, the GEARS study included participants according to the WM diagnosis of COPD in addition to the CM diagnosis of lung qi deficiency or spleen qi deficiency, or both. The intervention in this study was a 
herb used to treat lung qi deficiency or spleen qi deficiency, or both [14]. The WM and CM diagnoses enabled the translation of the outcomes into clinical practices.

The RCTs that require specialist testing and diagnostic equipment to confirm a diagnosis may be better suited to the hospital setting. Through negotiation with relevant hospital departments, access to testing and equipment may facilitate trial recruitment and reduce costs. Diagnostic X-ray was often required in the MEDACT study to exclude injuries caused by fracture. In the GEARS study, lung function testing was undertaken using spirometry, hospital pathology laboratories performed full blood examinations, including tests of liver and kidney function and trial medications were dispensed from the hospital pharmacy.

\section{Personnel and resources}

Conducting a high standard clinical trial requires a dedicated and experienced research team. Skilled investigators and trial coordinators are frequently employed by hospital research departments and are essential in a successful study [16]. The culture of an organisation and their openness to CM theory will aid in the conduct of the trial and contribute to recruitment success [17]. A hospital setting would be counterproductive if researchers in hospitals are not enthusiastic in CM research projects.

In the MEDACT study, ED doctors confirmed eligibility of participants then qualified acupuncturists delivered the acupuncture treatments. In the GEARS study, a qualified $\mathrm{CM}$ practitioner was employed during the screening process to ensure the participants met the pre-specified CM diagnostic criteria. All other activities, including randomisation, were completed by a clinical trial coordinator employed by the hospitals.

Study sites should be accessible to trial participants and responsive to queries if problems arise. Hospitals often have an established research culture and can offer a setting that improves the running of trials by providing the facilities required to ensure 'Good Clinical Practice' (GCP). Other advantages include the availability of facilities (e.g., radiology and pathology equipment) and trained specialized staff. Hospital based pharmacy departments are often a convenient location for storing and dispensing study medications, which assist in maintaining the integrity of blinding. Availability of these facilities was crucial to the conduct of both MEDACT and GEARS.

Hospitals have the potential to be more expensive than universities or clinic settings for conducting RCTs when taking into account the costs of site-set up, ongoing fees, personnel, per-recruitment charges and the legal costs of clinical trial agreements. This was found to be the case for the GEARS study. The study incurred approximately $\$ 5,500$ (Australian dollars) of additional hospital fees per site. This included ethics, site-set up, administration fees, ongoing fees, and additional per-recruitment charges of up to $\$ 250$. The MEDACT study also incurred similar costs.

\section{Participant recruitment}

Recruitment and retention of participants in clinical trials can be challenging due to trial-specific demands, the availability of patients, the preferences of patients and informed consent [18]. Individuals may participate in CM studies just as willingly as WM studies [19]; however, there is a dearth of research evaluating how the trial site might influence study participation.

The attitudes of potential participants towards CM clinical trials also influence recruitment $[17,20]$. Positive attitudes in recruitment for $\mathrm{CM}$ clinical trials include: (1) CM is considered safer and less invasive than WM; (2) CM treatments may help to reduce dependence on conventional medications; and (3) the willingness of participants to try new interventions at little cost or harm to themselves. By contrast, barriers to recruitment include: (1) doubt about the effectiveness of treatments; (2) lack of knowledge of how CM may work; (3) participants are not willing to change their current health care options and reluctant for randomisation when CM treatments are readily available in the community.

In the MEDACT study, patients were approached for screening soon after triage by the ED nurse. An early approach appeared to improve the chances of participation. The steady flow of patients through the ED combined with the motivation of these patients to obtain pain relief enabled satisfactory recruitment within a relatively short period. Little advertisement or incentive saved costs and enabled staff to concentrate on the ED during peak times in order to maximise recruitment.

A different recruitment strategy was used for the GEARS study as patients with stage II COPD were more likely to be outpatients. The recruitment strategy included media promotion, advertising in clinic rooms, and physician referrals. As this trial has not been completed, we have not yet evaluated the recruitment strategy.

\section{Conclusion}

The appropriateness of conducting a hospital-based CM clinical trial depends on the requirements for the clinical setting. We identified several key elements to consider before conducting a CM RCT in a hospital, including protocol design; personnel and resources; and participant recruitment. 


\section{Abbreviations}

ANZCTR: Australian and New Zealand clinical trials registry; CM: Chinese medicine; COPD: Chronic obstructive pulmonary disease; EBM: Evidencebased medicine; ED: Emergency department; GCP: Good clinical practice; GEARS: Ginseng extract and respiratory symptoms; MEDACT: Multiple emergency department acupuncture trial; RCT: Randomized controlled trial; WM: Western medicine.

\section{Competing interests}

The authors declare that they have no competing interests.

\section{Authors' contributions}

JS, SP and CX determined the manuscript scope and information. JS, SP, MC, $A Z$ and $C X$ wrote the manuscript. All authors read and approved the final version of the manuscript.

\section{Acknowledgements}

This work was partially supported by a project grant from the National Health \& Medical Research Council (Project Grant Number: 616609), and an International Research Fund Grant from the Guangdong Provincial Academy of Chinese Medical Sciences, Guangdong Provincial Hospital of Chinese Medicine, China.

Received: 15 October 2012 Accepted: 14 February 2013 Published: 16 February 2013

\section{References}

1. A European approach to non-conventional medicines. France: Social, Health and Family Affairs Committee, Parliamentary Assembly, Council of Europe; 1999

2. Barnes PM, Powell-Griner E, MCFann K, Nahin RL: Complementary and alternative medicine use among adults: United States, 2002. Adv Data 2004, 343:1-19.

3. Australian Social Trends 2008. Article: Complementary therapies, cat no. 4102.0. Canberra, Australia: Australian Bureau of Statistics; 2008.

4. Waddell $G$, Feder $G$, McIntosh A, Lewis M, Hutchinson A: Low back pain evidence review London. J Manual and Manipulative Ther 1998, 6:151-153.

5. Acupuncture: NIH Consensus Development Conference Statements Online 1997 Nov 3-5; 15: 1-34: http://consensus.nih.gov/1997/1997acupuncture107html.htm.

6. Silvert M: Acupuncture wins BMA approval. Br Med J 2000, 321(7252):11.

7. 'Complementary medicine gets a boost' Press release by Senator the Hon. Jan McLucas. 30 March 2008. http://www.nhmrc.gov.au/media/releases/2008/ complementary-medicine-gets-boost.

8. Chinese Medicine Board of Australia: Australian Health Practitioners Regulation Agency 2012. http://www.chinesemedicineboard.gov.au/.

9. Cohen MM, Penman S, Pirotta M, Da Costa C: The integration of complementary therapies in Australian general practice: Results of a national survey. J Altern Complement Med 2005, 11:995-1004.

10. WHO: Handbook for good clinical practice (GCP): guidelines for implementation. France: World Health Organisation; 2005.

11. Shea JL: Applying evidence-based medicine to traditional Chinese medicine: debate and strategy. J Altern Complement Med 2006, 12:255-263.

12. Clinical Trials Action Group: Clinically competitive: Boosting the business of clinical trials in Australia. Australia: Canberra; 2011.

13. Cohen M, Parker S, Taylor D, Smit DV, Ben-Meir M, Cameron P, Xue CC Acupuncture as analgesia for low back pain, ankle sprain and migraine in emergency departments: Study protocol for a randomized controlled trial. Trials 2011, 12:241.

14. Xue CC, Shergis JL, Zhang AL, Worsnop C, Fong H, Story D, Da Costa C, Thien F: Panax ginseng C.A Meyer root extract for moderate Chronic Obstructive Pulmonary Disease (COPD): study protocol for a randomized controlled trial. Trials 2011, 12:164.

15. Nahin RL, Straus SE: Research into complementary and alternative medicine: Problems and potential. Br Med J 2001, 322(7279):161-164

16. Campbell MK, Snowdon C, Francis D, Elbourne D, McDonald AM, Knights R, Entwistle V, Garcia J, Roberts I, Grant A: Recruitment to randomized trials: Strategies for trial enrolment and participation study. The STEPS study. Health Technol Assess 2007, 11:iii-72.

17. Smith CA, Coyle ME: Recruitment and implementation strategies in randomized controlled trials of acupuncture and herbal medicine in women's health. Complement Ther Med 2006, 14:81-86.
18. Ross S, Grant A, Counsell C, Gillespie W, Russell I, Prescott R: Barriers to Participation in Randomized Controlled Trials: A Systematic Review. J Clin Epidemiol 1999, 52:1143-1156.

19. Sood A, Prasad K, Wahner-Roedler D, Cha S: Patients' preferences about participation in clinical trials studying complementary and alternative medicine. Altern Ther Health Med 2011, 17:36-42.

20. Schneider J, Vuckovic N, DeBar L: Willingness to participate in complementary and alternative medicine clinical trials among patients with craniofacial disorders. J Altern Complement Med 2003, 9:389-401.

doi:10.1186/1749-8546-8-3

Cite this article as: Shergis et al:: Key considerations for conducting

Chinese medicine clinical trials in hospitals. Chinese Medicine 2013 8:3.

\section{Submit your next manuscript to BioMed Central and take full advantage of:}

- Convenient online submission

- Thorough peer review

- No space constraints or color figure charges

- Immediate publication on acceptance

- Inclusion in PubMed, CAS, Scopus and Google Scholar

- Research which is freely available for redistribution

Submit your manuscript at www.biomedcentral.com/submit
C) Biomed Central 